\title{
Studies on Hydration Swelling and Bound Water Type of Sodium- and Polymer-Modified Calcium Bentonite
}

\author{
Yong Wan $\mathbb{D}^{1,2}$ Dongdong Guo $\mathbb{D}^{1,2}$ Xinminnan Hui, ${ }^{1,2}$ Lei Liu $\mathbb{D}^{1},{ }^{1}$ and Yuan Yao ${ }^{3}$ \\ ${ }^{1}$ State Key Laboratory of Geomechanics and Geotechnical Engineering, Institute of Rock and Soil Mechanics, Chinese Academy \\ of Sciences, Wuhan 430071, China \\ ${ }^{2}$ University of Chinese Academy of Sciences, Beijing 100049, China \\ ${ }^{3}$ Wuhan Environment Investment \& Development Co., Ltd, Wuhan 430019, China
}

Correspondence should be addressed to Yong Wan; ywan@whrsm.ac.cn and Dongdong Guo; guodongdong18@mails.ucas.ac.cn

Received 30 May 2020; Revised 20 July 2020; Accepted 5 August 2020; Published 9 October 2020

Academic Editor: Adam Kiersnowski

Copyright (c) 2020 Yong Wan et al. This is an open access article distributed under the Creative Commons Attribution License, which permits unrestricted use, distribution, and reproduction in any medium, provided the original work is properly cited.

\begin{abstract}
Modified calcium bentonite (Ca-bentonite) is extensively used in engineered barrier systems (EBSs) for municipal and industrial disposal sites due to its high swelling potential and low hydraulic conductivity. However, few studies have focused on the micromechanism of hydration and swelling under the effect of inorganic chemical solution. In this study, free swell index (FSI) and the type and content of modified Ca-bentonite bound water under the inorganic chemical solution were quantitatively studied by using the free swell test and nuclear magnetic resonance (NMR). According to the results, modification of sodium and polymer significantly increases the FSI of Ca-bentonite, bringing it close to that of natural sodium bentonite. In addition, the chemical stability of polymer-modified bentonite is significantly higher than that of sodium-modified bentonite but less than that of natural Na-bentonite. The FSI of modified Ca-bentonite decreases with the increase of cation valence and ionic strength. $T_{2}$ distribution curves of the two types of modified bentonite are three-peak curves. With the increase of ionic strength, the content of total water and permeated hydrated water (accounting for 69\%-95\%) in bentonite decreases gradually, whereas the surface hydration water (accounting for 2\%-31\%) and free water content (accounting for 0-15\%) increase. A uniform linear relationship exists between the FSI and corresponding total peak area of NMR (independent of ion valence, concentration, and bentonite type). Furthermore, a linear relationship exists between the FSI of the same type of bentonite and the $T_{2}$ relaxation time. Research results can provide data and theoretical basis for quantitative analysis and mechanism of the hydration swelling of bentonite.
\end{abstract}

\section{Introduction}

Bentonite, which mainly consists of montmorillonite clay minerals, is widely used in engineered barrier systems (EBSs) due to its low hydraulic conductivity that restricts pollutant migration and minimizes the risk of groundwater contamination. Sand-bentonite mixtures are typically used as buffer/backfill materials for the disposal of high-level radioactive pollutants; soil-bentonite vertical cutoff walls and geosynthetic clay liners (GCLs) are mostly used in the EBS for municipal and industrial disposal sites [1-3]. The low permeability of the bentonite mixture is mainly determined by the swelling ability of the bentonite. The reason is that its main mineral component, montmorillonite, has strong hydration and swelling properties, which cause filling of the pores between the soil particles, as well as narrowing and curving of the permeation channels, thereby reducing permeability to achieve the function of blocking and preventing the migration of pollutants.

The hydration swelling mechanism of bentonite can be explained through the theory of diffusion double layer. The adsorbed cations on the surface of bentonite are diffused to equalize concentration throughout the pore fluid; the charged surface and distributed charge in the adjacent phase are together called diffuse double layer, as shown in Figure 1, which includes Stern layer and Gouy-Chapman diffusion layers on the surface of clay particles [4]. The swelling ability of bentonite is positively related to the thickness of the 


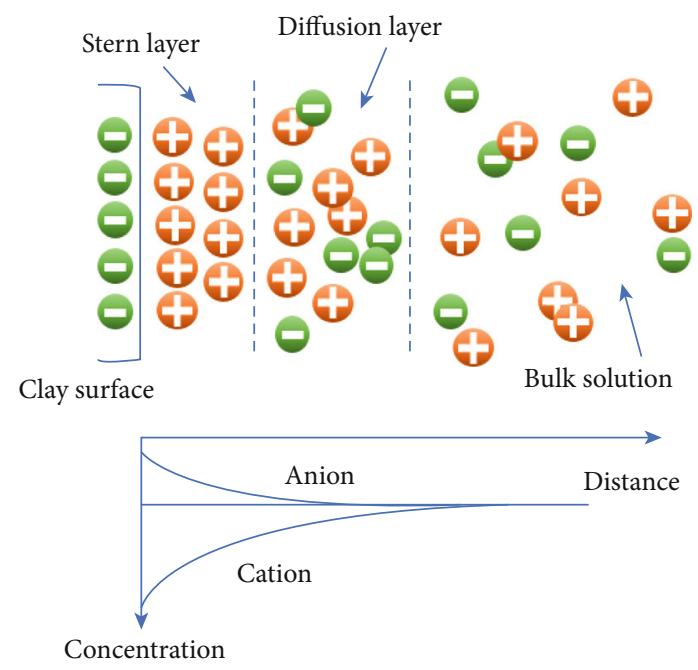

FIgURE 1: Stern electrical diffusion double layer model.

diffusion double layer, whereas the type of bentonite (sodium-based or calcium-based) and ionic strength of the pore fluid are the two key factors that determine the thickness and swelling ability of the diffusion double layer. In the process of blocking the pollutant migration, the high concentration of cations in the pollutants greatly compresses the thickness of the double layer of the bentonite, causing the reunion of the bentonite particles, thereby reducing the swelling ability of the bentonite and increasing its permeability. As a result, the performance of bentonite in blocking pollutants is reduced.

Studies on the evolution of the swelling and permeability performance of bentonite under the effect of inorganic chemical solution have always been an important research focus in environmental geotechnical engineering. Many previous studies have shown that under the chemical attack of low ionic strength solutions and leachate, the hydration cations between the bentonite layers exchange with the ions in the solution. The high-valence cations in the solution can replace the sodium ions between the interlayers of bentonite, thereby reducing the bentonite swelling and resulting in the failure of GCL permeability performance $[3,5,6]$. Shackelford et al. [7] found that containing high-concentration monovalent cations and low-concentration divalent cations can cause GCL hydraulic conductivity to increase significantly by at least one order of magnitude. Jo et al. [8] conducted the influence of $\mathrm{NaCl}, \mathrm{KCl}$, and $\mathrm{CaCl}_{2}$ solutions on the long-term permeability characteristics of GCL and investigated the variation law of hydraulic conductivity. Clay with greater content of sodium montmorillonite (Na-MMT) is more susceptible to the penetration of inorganic solutions, and the chemical solutions that contain different species of cations have varying influences on clay, as confirmed by Lee and Shackelford [9]. Di Maio [10] exposed the bentonite sample to saturated $\mathrm{NaCl}, \mathrm{KCl}$, or $\mathrm{CaCl}_{2}$ solutions and observed inward diffusion produced ion exchange in the cases of $\mathrm{KCl}$ and $\mathrm{CaCl}_{2}$. Kolstad and Benson et al. [11-13] further studied the effect of multispecies inorganic solutions containing polyvalent cations on the swelling and permeability properties of the sodium-bentonite from GCL. The high cation valence further reduces the swelling of bentonite, increases the hydraulic conductivity, and improves the permeability. A strong relationship was observed between permeability and free swell.

When clay minerals and inorganic solutions interact, causing changes in their structure leads to differences in hydration swelling behavior [14]. The difference of swelling ability is essentially related to the change of bound water content and type under different hydration states on the surface of bentonite particles. When bentonite particles interact with chemical solutions, the hydrated cation in the solution is adsorbed to the surface of the bentonite particles to maintain the electrical neutrality. According to the adsorption force of the water molecules by the bentonite, the interlayer water can be divided from the inside out into surface-hydrated water (inside the Stern layer, solid), permeated hydrated water (inside the Gouy-Chapman diffusion layer, semisolid), and free water. Water adsorbed by clay is known to cause clay swelling to a certain extent. Phase changes of the water present in soils play an important role in the swelling process [15]. Mojid and Cho [16] estimated the thickness $(t)$ of the fully developed diffuse double layer (DDL) from water content at the fully developed state of the DDLs, which agrees well with the result calculated using the method of Schofield [17], and proved that the critical water content is a direct function of the specific surface area of the clay and the thickness of the fully developed DDLs.

Over the last decade, various experimental approaches to study the existence state and evolution law of water in soil on a microscale, such as mercury intrusion porosimetry, scanning electron microscopy, nitrogen adsorption, and X-ray visualization, have been developed and widely used in soil microstructure testing to reveal the inherent nature of changes in the soil macrophysical and mechanical properties from a microscopic level [15, 18-20]. However, the preceding microtechnical testing methods require soil drying, which is difficult to apply in the investigation of micromechanisms of bentonite hydration and swelling. The NMR is a quantitative testing method that can rapidly, nondestructively, and accurately determine the content of various types of water in soil pores. Wang et al. [21] used solid-state $1 \mathrm{H}$ magic-angle spinning NMR spectroscopy to investigate molecular-level interactions of the interlayer water near the montmorillonite surfaces on commercial bentonite; theoretical calculation showed that two types of interaction in the interlayer space of montmorillonite may occur: water-water and cation-water interaction. The waterwater interaction formed loosely bound water and the water-cation interaction formed tightly bound water near the montmorillonite surfaces. The water-water interaction was dominant at relatively high water content, and the cation-water interaction was dominant at relatively low water content. Ohkubo et al. [22] investigated the compacted saturated bentonites using the NMR technique, assuming the relaxation time with a 3-hydrated layer, which was used as a threshold to justify whether the bulk-like water (pore water) is, and evaluated the proportion of bulk-like water to total water. The proportion of bulk-like water obtained from continuous distribution analysis of relaxation time was in 
agreement with previous XRD results. Xie et al. [15] found that the experiment results for the cationic interlayer water and interlayer surface water measured using low-field nuclear magnetic resonance (LF-NMR) were consistent with the thermogravimetric analysis (TGA) results; the adsorbed water on Na-MMT of the first step was the cationic interlayer water, the second step was the interlayer surface water, and the third step was the sum of the part of the interlayer surface water and free water. In this experiment, the bentonite swelled and dispersed in the solution. TGA of the slurry sample obtained by centrifugation produced great errors, whereas the NMR technique accurately completed the study. Tian et al. $[23,24]$ integrated proton NMR spin-spin relaxation time $\left(T_{2}\right)$ distribution measurement with a Tempe apparatus to characterize the hydraulic processes of unsaturated soils, shedding insights into the microscopic mechanisms of pore water distribution and migration in the soil during hydraulic cycles, and further determined the pore size distribution of the soil. The new procedure was validated by comparing the new result with the measurement through the MIP.

The type of cation between interlayers is the key factor to determine the bentonite swelling ability. At present, natural Na-bentonite is the most commonly used element in environmental geotechnical engineering for containment barriers, whereas Ca-bentonite is difficult to use directly because of its extremely poor properties such as swelling, cation exchange ability, dispersibility in solution, and thermal stability [25]. China has abundant reserves of bentonite, but the natural Na-bentonite only accounts for approximately $10 \%$, and most of the reserves are Ca-bentonite. To improve the swelling performance of Ca-bentonite, this material is commonly modified with sodium or polymer, but research on the chemical stability of the modified bentonite interacting with pollutants is rarely reported. Therefore, the present study takes sodium- and polymer-modified Ca-bentonite as the research object, discussing the attenuation law of the modified Ca-bentonite swelling under different concentrations and multivalence cations, and combining with the LFNMR technique to quantitatively evaluate the changes of different water types in hydration bentonite. The research results provide parameter thresholds and theoretical support for the application of safety analysis of modified bentonite in pollution barrier systems.

\section{Materials and Methods}

2.1. Materials. In this study, two typical commercially modified varieties of $\mathrm{Ca}$-bentonite were selected, of which bentonite 1 was sodium-modified Ca-bentonite, and bentonite 2 was polymer-modified Ca-bentonite adding anionic polyacrylamide (APAM) and cationic polyacrylamide (CPAM). Information on the two types of polymer modifier are listed in Table 1. The basic properties of the two kinds of modified Ca-bentonite are presented in Table 2. The XRD spectra of bentonite 1 and 2 are shown in Figure 2. The basal spacing of the sodium-modified Ca-bentonite (B1) was $1.2897 \mathrm{~nm}$, and the polymer-modified Ca-bentonite (B2) had a larger basal spacing of $1.4949 \mathrm{~nm}$. These results could be attributed
TABLE 1: Physical properties of polymer modifier.

\begin{tabular}{lccc}
\hline & Preparation method & Ionic degree & Concentration \\
\hline CPAM & Solution polymerization & $8.91 \mathrm{~mol} \%$ & $2.7 \mathrm{wt} \%$ \\
APAM & Solution polymerization & $4.1 \mathrm{~mol} \%$ & $3.5 \mathrm{wt} \%$ \\
\hline
\end{tabular}

TABLE 2: Physical properties of tested bentonite.

\begin{tabular}{ccccccc}
\hline & $\begin{array}{c}\text { CEC } \\
(\mathrm{meq} / 100 \mathrm{~g})\end{array}$ & Gs & $\begin{array}{c}\text { Liquidity } \\
\text { limit }\left(\omega_{\mathrm{L}}\right)\end{array}$ & $\begin{array}{c}\text { Plasticity } \\
\text { limit }\left(\omega_{\mathrm{P}}\right)\end{array}$ & $\begin{array}{c}\text { SSA } \\
\left(\mathrm{m}^{2} / \mathrm{g}\right)\end{array}$ & $\begin{array}{c}\text { FSI } \\
(\mathrm{ml} / 2 \mathrm{~g})\end{array}$ \\
\hline B1 & 73.5 & 2.71 & 353 & 86 & 379 & 26 \\
B2 & 65.2 & 2.63 & 289 & 59 & 267 & 25 \\
\hline
\end{tabular}

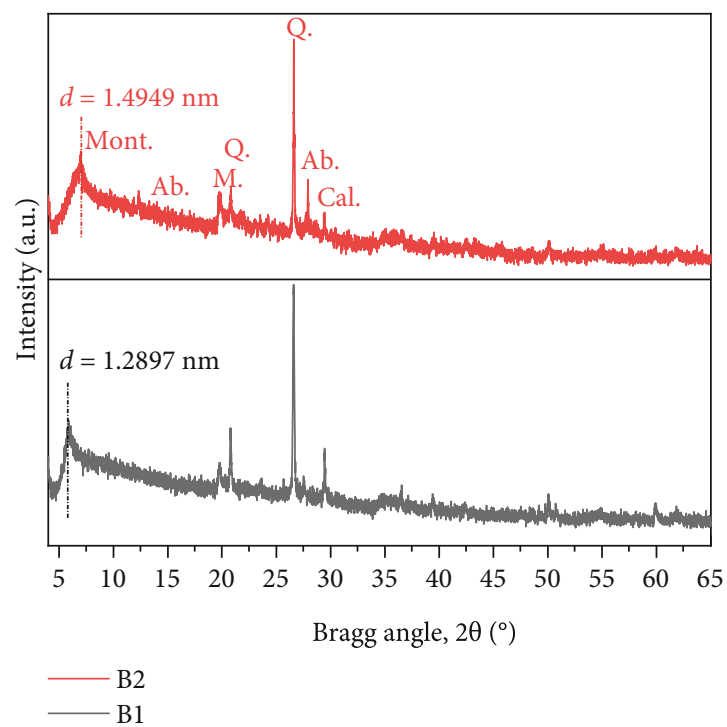

FIgURE 2: X-ray diffraction patterns of soils used in this study: B1 and $\mathrm{B} 2$.

to the replacement of $\mathrm{Ca}^{2+}$ by PAM in the organic intercalation reaction of $\mathrm{B} 2$, and the basal spacing of bentonite would increase for the larger size of organic molecules.

In previous research $[7,11,12], \mathrm{NaCl}$ and $\mathrm{CaCl}_{2}$ were selected to simulate the solution containing monovalent and divalent cations. The present study mainly considered the influence of monovalent and divalent cations on the chemical stability of bentonite. Here, the ionic strength was set as $0,10,50,100$, and $500 \mathrm{mM}$. The $\mathrm{AR} \mathrm{NaCl}$ and $\mathrm{CaCl}_{2}$ for the test were purchased from Sinopharm Chemical Reagent Co. Ltd. (China). The conductivity of deionized water used in the experiment was $0.055 \mu \mathrm{S} / \mathrm{cm}$.

\subsection{Methods}

2.2.1. Free Swell. The free swell tests were conducted according to ASTM D5890 [26]. The bentonite was ground into a fine powder with a mortar and pestle. The samples were sieved from No. $200(75 \mu \mathrm{m})$, air dried for $24 \mathrm{~h}$, and then stored in an airtight container prior to testing. A $100 \mathrm{ml}$ graduated cylinder, accurate to $\pm 0.5 \mathrm{ml}$, was used to inject a solution configured according to the predetermined ion 
concentration up to $90 \mathrm{ml}$. Then, $2 \mathrm{~g}$ of sieved bentonite was added to the measuring cylinder slowly in increments of $0.1 \mathrm{~g}$. The time interval between the two additions is not less than $10 \mathrm{~min}$. Then, solution was further added to the measuring cylinder until the volume reached $100 \mathrm{ml}$, and the flow rushed the particles attached to the wall into the solution. If present air or water separation in the column, the cylinder is gently tipped at a $45^{\circ}$ angle and rolled slowly to homogenize the settled clay mineral mass. The graduated cylinder with the hydrating clay mineral was left undisturbed for a minimum of $16 \mathrm{~h}$ before recording the volume of the hydrated clay mass and its temperature was recorded. After $16 \mathrm{~h}$ of exposure, free swell index (FSI) corresponded to the bentonite swelling height measured in the cylinder $(\mathrm{ml} / 2 \mathrm{~g})$. This test was conducted in triplicate, and the mean result is presented in the following discussion.

2.2.2. NMR. After the free swell test, the upper free water in the graduated cylinder was drained. Then, the specimen saturated with the chemical solution (DI water, $\mathrm{NaCl}$, and $\mathrm{CaCl}_{2}$ ) in the graduated cylinder was moved into the sample tube of the NMR apparatus, where the NMR measurement was conducted. The NMR apparatus used in the experiments were developed by Niumag Corp. (China). To generate a stable magnetic field, the temperature of the magnet unit was held at $32^{\circ} \mathrm{C}$, with a variation of $0.01^{\circ} \mathrm{C}$. The NMR technique uses a proton group with a spin magnetic moment located in a uniform magnetic field (main magnetic field) to lose balance after deflection under the action of interfering radiofrequency magnetic fields. When the radiofrequency interference stops, the proton group returns from an unbalanced state to a balanced state. To determine the nuclear magnetic signal free attenuation (FID) curve, the shape of the FID curve is related to the proton lateral relaxation time $\left(T_{2}\right)$. The theoretical analysis [23] shows that the transverse relaxation time of the pore fluid $\left(T_{2}\right)$ and pore radius $(R)$ is expressed in the following relationship:

$$
\frac{1}{T_{2}}=\rho_{2} \frac{2}{R},
$$

where $\rho_{2}$ is the $T_{2}$ surface relaxivity. Although $\rho_{2}$ varies with the mineralogical composition of the soil, it is a constant for a specified soil and independent of temperature and pressure.

Equation (1) implies that $T_{2}$ of the pore fluid is proportional to the pore radius $R$, that is, in a larger pore, more time is needed for the water molecules to reach equilibrium and the $T_{2}$ of adsorbed water or smaller pore is less than that of the larger pore. Based on this theory, the $T_{2}$ relaxation time distribution curve of the specimen can directly reflect the pore fluid distribution in the geotechnical medium. In the following, the area under the $T_{2}$ distribution curve is called "peak area" for convenience (as defined in [27]), which represents the population of water molecules, indicating the amount of water content in the bentonite specimen [24]. In Figure 3, the conversion relationship between the peak area of the nuclear magnetic signal and water content is shown.

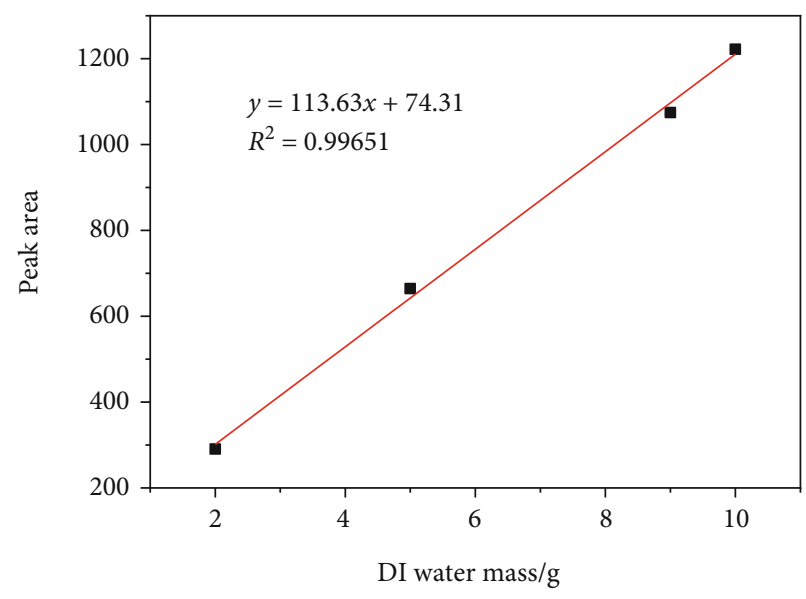

FIgURE 3: The conversion relationship between peak area and water content in bentonite. Line is the linear fit.

\section{Results}

3.1. Free Swell. The results of the FSI test in different ionic strength solutions are shown in Figure 4. Sodium modification and polymer modification significantly improved the FSI of Ca-bentonite (the FSI of natural Ca-bentonite in DI water is only approximately $10 \mathrm{ml} / 2 \mathrm{~g}$ ). In DI water, the FSIs of the two kinds of modified Ca-bentonite are relatively close (B1 is 26.0; B2 is 25.0). With the increase of ionic strength, the FSI of bentonite decreases accordingly. When the ionic strength is close to $500 \mathrm{mM}$, the FSI of bentonite tends to be stable (B1 tends to approximately 5.0 and B2 tends to approximately 9.0). However, the sodium-modified $\mathrm{Ca}$ bentonite has poor chemical stability. As shown in Figure 4, the initial FSI of the sodium-modified bentonite is higher than that of the polymer-modified bentonite. As the ionic strength increases, the FSI of the sodium-modified bentonite decreases faster and the final stable FSI is lower. Therefore, we can conclude that the sodium-modified bentonite has a lower resistance to the erosion of chemical solutions and a weaker ability to maintain its high swelling capacity. At the same time, this problem can also be explained by the modification mechanism. The method of polymer modification is that the ionic polymer enters the montmorillonite interlayer because of its stronger affinity for the interlayer domain, so the ion exchange does not easily occur again, which may change the mineral structure and lead to the failure of the antiseepage function. Prolonged exposure of the modified Ca-bentonite to multispecies fluids disturbs the electronic equilibrium, causing a loss of hydraulic performance [28], and a similar conclusion is observed in this study. The effect of cation on the FSI of polymer-modified bentonite is less than that of sodium-modified bentonite. Under the same ionic strength, the effect of divalent cations on the FSI is significantly greater than that of monovalent cations for polymer-modified bentonite (although the difference of cation valence is not obvious for sodium-modified bentonite). The main reason is that the exchange capacity of divalent cations is greater than that of monovalent cations. As observed 

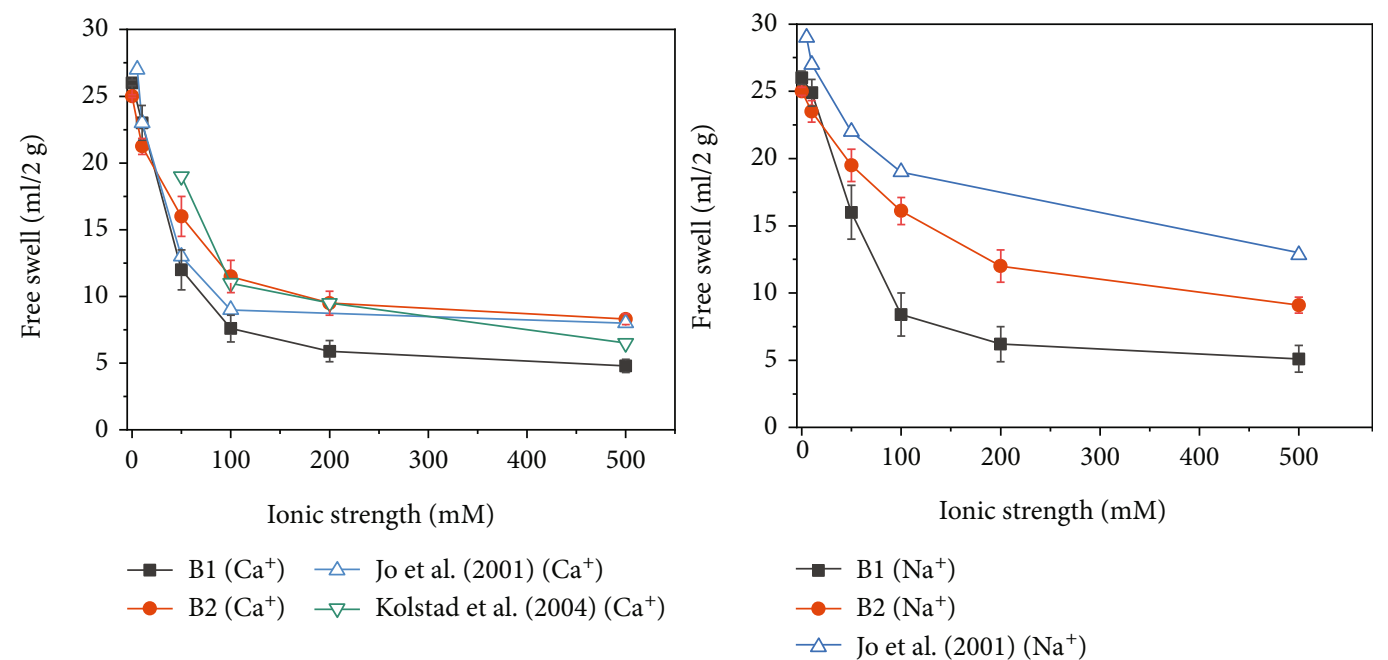

FIGURE 4: Free swell of bentonite as a function of different ionic strengths.
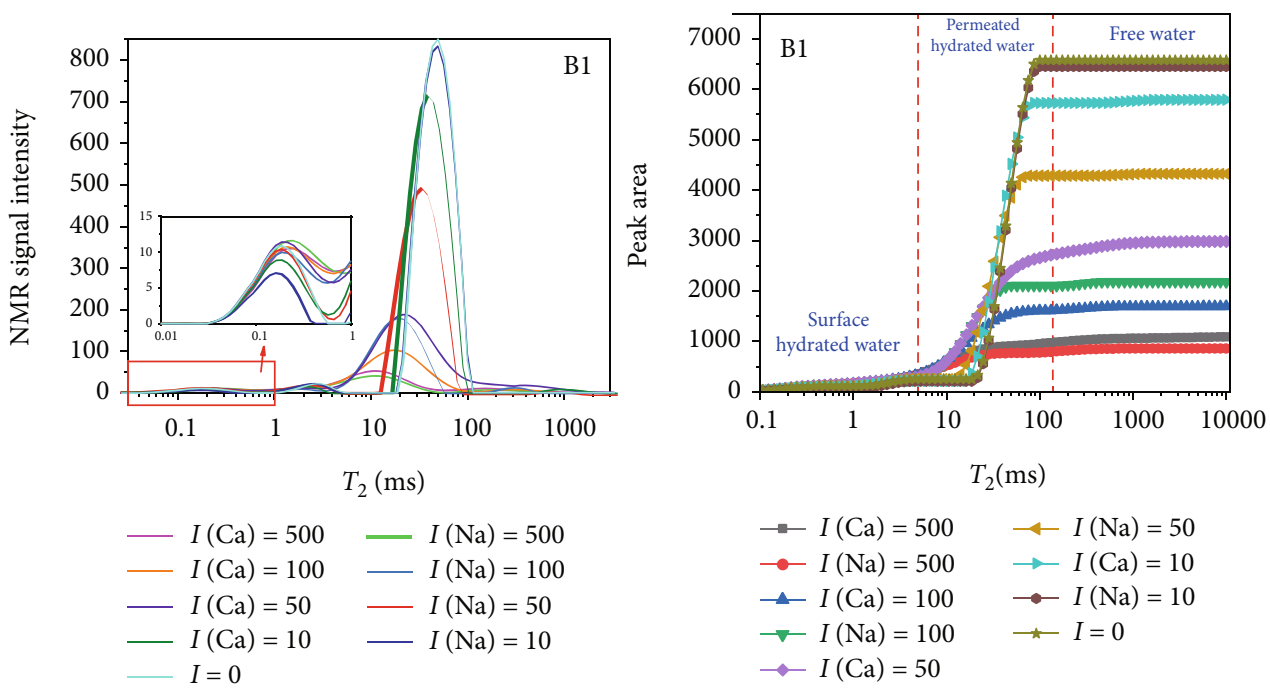

Figure 5: $T_{2}$ distribution curves and peak areas in $T_{2}$ distribution of sodium-modified Ca-bentonite B1 at different ionic strengths.

in Figure 4, the FSI of the sodium-modified bentonite does not change considerably in the $\mathrm{Ca}^{2+}$ and $\mathrm{Na}^{+}$solutions, but changes greatly in the polymer-modified bentonite. As the sodium-modified bentonite is obtained by exchanging the interlayer of bentonite with the cations in the permeate, $\mathrm{Ca}^{2+}$ and $\mathrm{Na}^{+}$remain between the interlayers of the sodium-modified bentonite, thereby limiting the amount of ion exchanges that occur in the solution, which results in a minimal effect on the bentonite structure. Compared with the natural Na-bentonite reported by Jo et al. (2001) and Kolstad et al. (2004) $[11,12]$, our study showed that the FSI of modified Ca-bentonite in DI water is close to that of natural Na-bentonite. However, in the monovalent $\mathrm{Na}^{+}$solution, as the ionic strength increases, the FSI attenuation rate of modified Ca-bentonite is significantly greater than that of natural Na-bentonite, whereas in the divalent $\mathrm{Ca}^{+}$solution, no obvious difference is observed between the FSI attenuation rates of the aforementioned two types of bentonite.
3.2. NMR. On the basis of the NMR tests, the $T_{2}$ distribution curves and peak areas of the nuclear magnetic signal in $T_{2}$ distribution of sodium-modified and polymer-modified Cabentonite at different cation species and ionic strength were obtained, as shown in Figures 5 and 6. The $T_{2}$ distribution curves of the two types of modified Ca-bentonite are threepeak curves, which, respectively, correspond to three types of bound water: surface hydrated (tightly bound water), permeated hydrated (loosely bound water), and free water. Among them, the permeated hydrated water (with the main peak in $T_{2}$ distribution, the $T_{2}$ of $\mathrm{B} 1$ in the permeated hydrated state centers around $1-100 \mathrm{~ms}$, and the $T_{2}$ of $\mathrm{B} 2$ in the permeated hydrated state centers around 5-150 ms) is the main type of bound water in bentonite. As the concentration of cations increases, the peak value and area of the middle main peak gradually decrease, and the $T_{2}$ corresponding to the peak value gradually decreases, as indicated in Figure 7. The main reason is that as the cation 

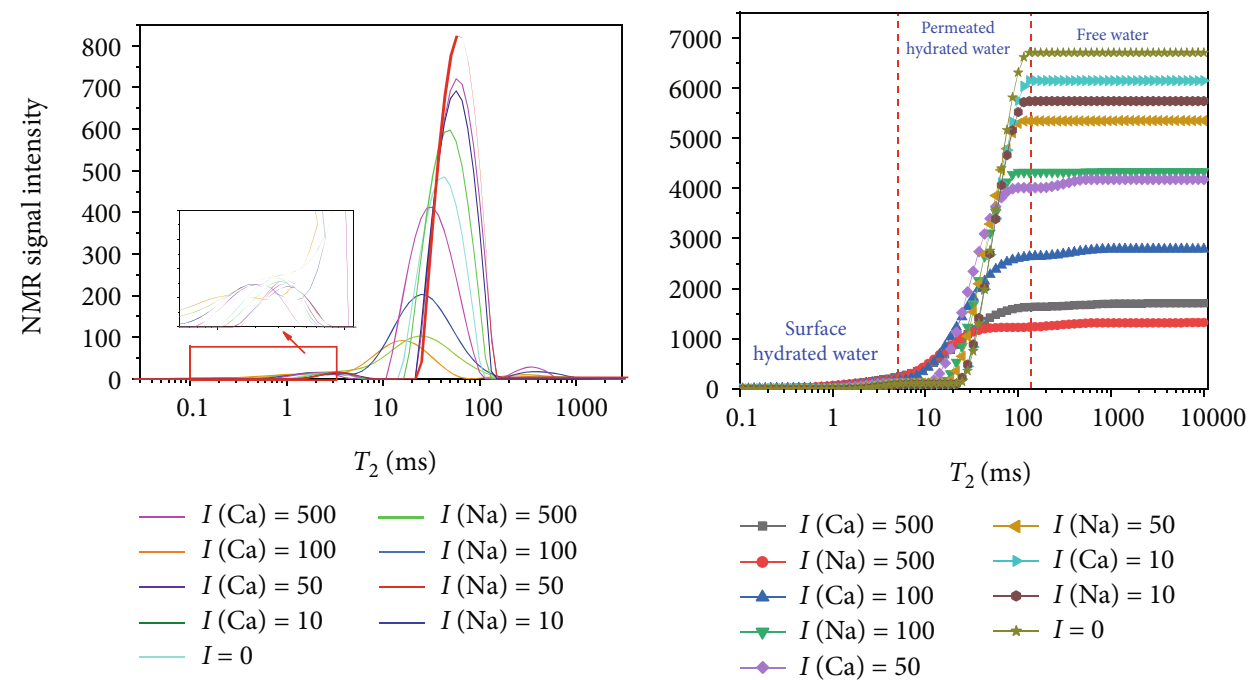

Figure 6: $T_{2}$ distribution curves and peak areas in $T_{2}$ distribution of polymer-modified Ca-bentonite B2 at different ionic strengths.

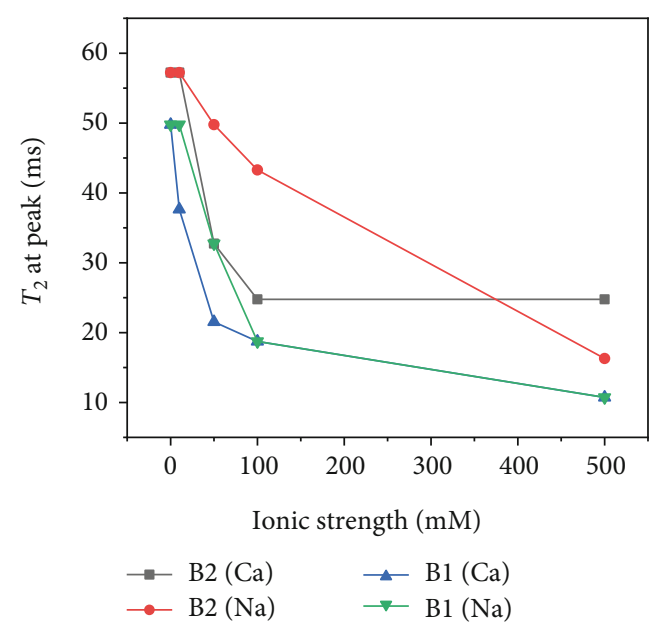

Figure 7: $T_{2}$ at peak as a function of ionic strength.

concentration increases, the double layer of bentonite is gradually compressed, the permeated hydrated water gradually decreases, the spacing between particles decreases, and the macroscopic performance shows that the FSI decreases.

\section{Discussion}

4.1. Diffusion Double Layer Theory of Surface Water Type of Bentonite. The mechanism of bentonite hydration swelling can be explained by the theory of diffusion double layer; the essence of which is the change of water content among different water types on the surface of bentonite particles [14]. When bentonite particles interact with chemical solutions, the hydrated cation in the solution is adsorbed to the surface of the bentonite particles to maintain the electrical neutrality. According to the adsorption force of the water molecules by the bentonite, the water content can be divided from the inside out into surface-hydrated water (inside the stern layer, solid), permeated hydrated water (inside the GouyChapman diffusion layer, semi-solid), and free water.
The FSI of bentonite is positively correlated with the thickness of the DDL on the surface of bentonite. According to the theory of diffusion double layer, the thickness of the double layer can be calculated by Equations (2)-(6). From Equations (2)-(5), the relationship shows that the thickness varies inversely with the valence and square root of the concentration and directly with the square root of the dielectric constant and temperature, with other factors remaining constant. As the long-range repulsive force between particles depends on the amount of overlap or interaction between opposing double layers, the probable influences on behavior that result from changes in the system composition can be estimated. In general, the thicker the diffuse layer is, the less the tendency for the particles in suspension to flocculate is and the higher the swelling pressure in bentonite is $[4,29]$.

$$
\begin{aligned}
\frac{1}{k} & =\sqrt{\frac{\varepsilon K T}{e^{2} \sum n_{i} z_{i}^{2}}} \\
n_{i} & =1000 M_{i} N_{\mathrm{A}}, \\
I & =\frac{1}{2} \sum M_{i} z_{i}^{2}, \\
\varepsilon & =\varepsilon_{0} D,
\end{aligned}
$$

where $e$ is the electronic charge $\left(1.60 \times 10^{-19} \mathrm{C}\right), n_{i}$ is the concentration of ions (ions $/ \mathrm{m}^{3}$ ) of type $i$ per unit volume, $z_{i}$ is the ionic valence of type $i, K$ is the Boltzmann constant $\left(1.38 \times 10^{-23} \mathrm{~J} \mathrm{~K}^{-1}\right), \varepsilon$ is the static permittivity of the pore solution $\left(\mathrm{C}^{2} \mathrm{~J}^{-1} \mathrm{~m}^{-1}\right), T$ is the temperature $(\mathrm{K}), M_{i}$ is the total molarity of cations (mol/L), $N_{\mathrm{A}}$ is Avogadro's constant $\left(6.022 \times 10^{23} \mathrm{~mol}^{-1}\right), I$ is the ionic strength $(\mathrm{mol} / \mathrm{L})$, and $1 / \kappa$ is the thickness of the double layer on the surface of soil particles.

To draw an approximate relationship, we assume that at room temperature, the ionic solution contains only monovalent ions $(D=70, T=298 \mathrm{~K})$, and the thickness of the electric double layer on the surface of the clay particles in the $\mathrm{NaCl}$ 


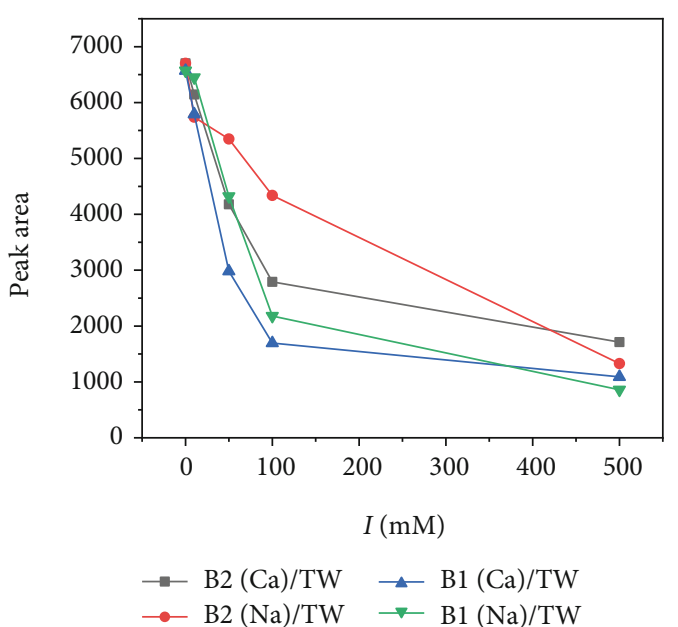

(a)

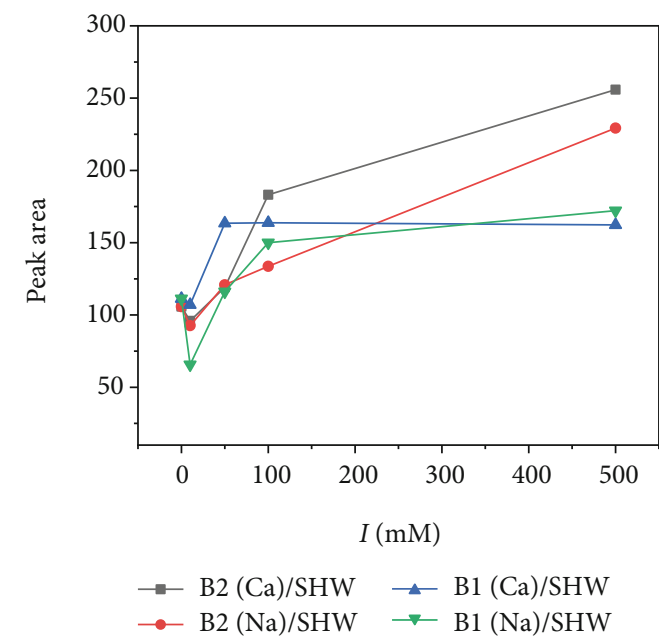

(c)

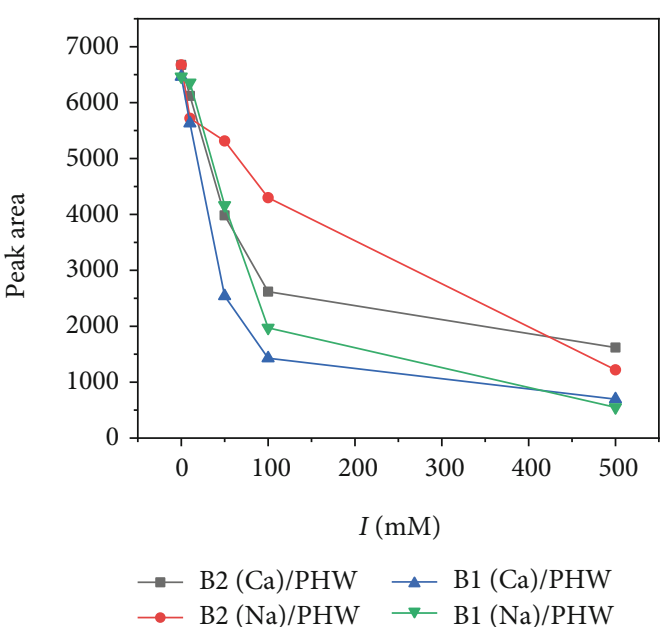

(b)

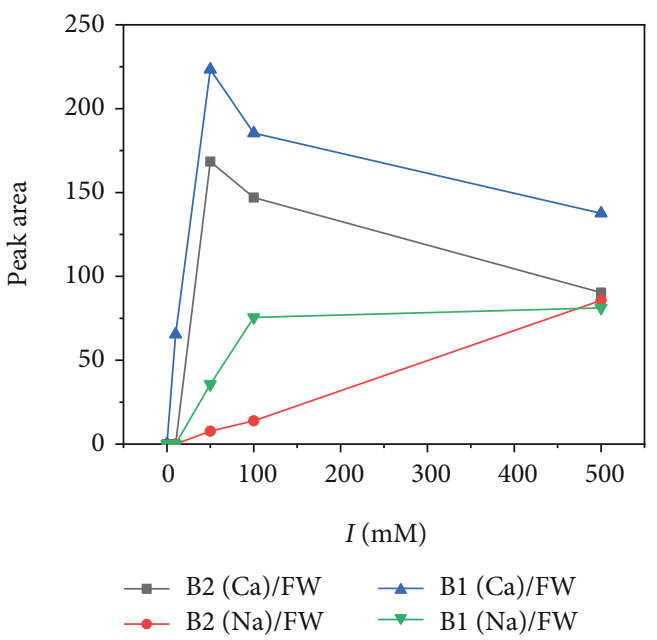

(d)

FIGURE 8: Relationship between ionic strength and peak area of different bound water types on surface of bentonite particles: (a) total water, (b) permeated hydration water, (c) surface hydration water, and (d) free water.

solution is derived as Equation (6). This equation explains that the thickness of the double layer is inversely proportional to the ionic strength (ion concentration and valence).

$$
\frac{1}{\kappa}=\frac{1}{3.4789 \sqrt{I}}(\mathrm{~nm}) .
$$

4.2. Quantitative Analysis of Surface Water Type of Bentonite. The relationship between the ionic strength and peak areas of different water types was determined from the peak areas of the nuclear magnetic signal and the classification of bound water types based on $T_{2}$ distribution shown in Figures 5 and 6. For B1 and B2, which interacted with solutions containing monovalent and divalent cations, the peak areas of the nuclear magnetic signal can be divided into total water, surface hydrated water, permeated hydrated water, and free water. As shown in Figures 8(a) and 8(b), permeated hydration water is the main water type, accounting for approximately $69 \%$ and $95 \%$. The total peak area has a similar variation law with permeated hydration water, which decreases gradually with the increase of ionic strength. The trend is similar to the change of FSI with ionic strength, and the reason is the compression of the diffuse double layer.

As shown in Figure 8(c), the surface hydration water content is relatively small; the interval of the peak area of $\mathrm{B} 1$ is $66-172$ and that of B2 is $84-233$. According to the relationship between peak area and water content in Figure 3, the corresponding interval mass of water is 0 $0.86 \mathrm{~g}$ and $0.09-1.40 \mathrm{~g}$, respectively, and the corresponding moisture content is $0 \%-43 \%$ and $5 \%-70 \%$. As the cation concentration increases, the surface hydration water content gradually increases. With the increase of ionic strength, more hydration cations enter the Stern layer and are adsorbed on the surface of the bentonite particles. At the same ionic strength, the effect of divalent ions on the Stern layer is more significant, which is consistent with the conclusion reported by Jo et al. [8]. Therefore, at the same ionic strength, surface hydration water content under the influence of divalent cations is higher than that of monovalent cations. 
As shown in Figure 8(d), with the increase of ionic strength, free water content gradually increases. At the same ionic strength, the free water content in B1 is significantly greater than that in $\mathrm{B} 2$, and the effect of $\mathrm{Ca}^{2+}$ solution is greater than that of $\mathrm{Na}^{+}$solution [30]. The dependence of the quantity of free water on ionic strength is different and, in some cases, nonmonotonic. The reason is that the water content of the modified bentonite under $\mathrm{Ca}^{2+}$ peaked at $50 \mathrm{mM}$, and then gradually decreased, because the initial high concentration of ionic solution caused the flocculation of the bentonite particles to form aggregates, forming a large number of interaggregation pores, so there is a proportional correlation and reaching a peak. Then, the diffusion double layer structure was compressed due to the cation exchange. The water content was also reduced, which was the main reason for the failure of the bentonite-based antiseepage system.

4.3. Relationship between FSI and Characteristic Parameters of NMR. According to the principle of the FSI test and NMR technique, the FSI is equal to the sum of the volume of $2 \mathrm{~g}$ bentonite particles and the volume of water. Based on the assumption that the density variation between various types of bentonite and bound water is ignored, a unique linear relationship is observed between the FSI and total peak area of the nuclear magnetic signal. In Figure 9, the linear fitting relationship between the FSI of bentonite and the total peak area of the nuclear magnetic signal under different working conditions (different cation valence and ionic strength) is shown. The results show that the linear correlation coefficient is 0.99 , and the fitting formula is shown as follows:

$$
\mathrm{TW}=284.5 \mathrm{FSI}-535.7 .
$$

In Figure 10, the linear fitting relationship between the FSI and the optimal $T_{2}$ (defined as the maximum $T_{2}$ at the main peak) is shown. For the same type of bentonite, an obvious linear relationship exists between the FSI of bentonite and the optimal $T_{2}$ under different working conditions (different cation valence state and ionic strength). The fitting results are shown in Equations (8) and (9). According to the quantitative relationship between $T_{2}$ and pore size (spacing between bentonite particles) in Equation (1), the optimal $T_{2}$ can be approximated as the average spacing between bentonite particles [30]. According to the theory of diffusion double layer, the reason for the hydration swelling of bentonite is the increase in the thickness of the diffusion double layer, that is, the increase in the spacing between particles. For the same type of hydrated bentonite, the larger the average porosity is, the thicker the double electric layer is, which means larger swelling capacity. For different types of hydrated bentonite in Figure 4, under the same ionic strength, the FSI of B2 $>$ B 1 , which shows that B2 has a larger swelling capacity under the same conditions. In Figure 10, under the same average pore size, the FSI of $\mathrm{B} 2<\mathrm{B} 1$. We verified that the expansion of interlayer domain is the main reason for the improved swelling capacity of B2 under the same working

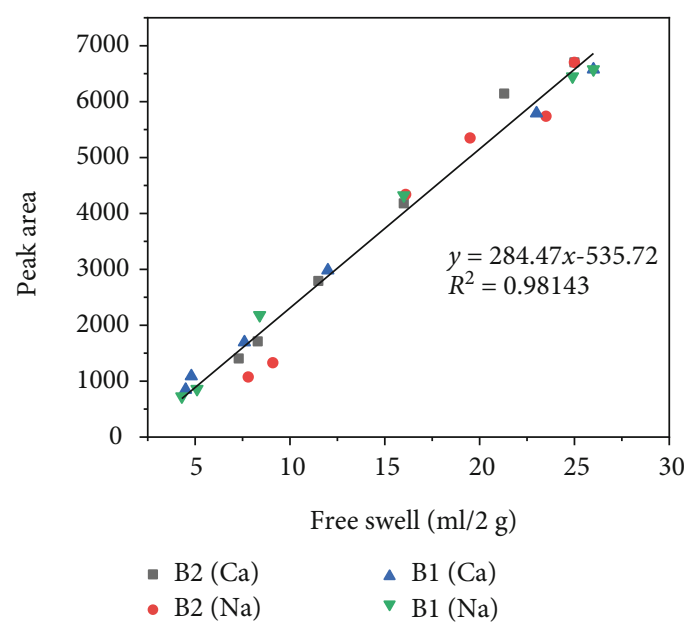

FIgURE 9: Linear fitting relationship between total peak area and FSI.

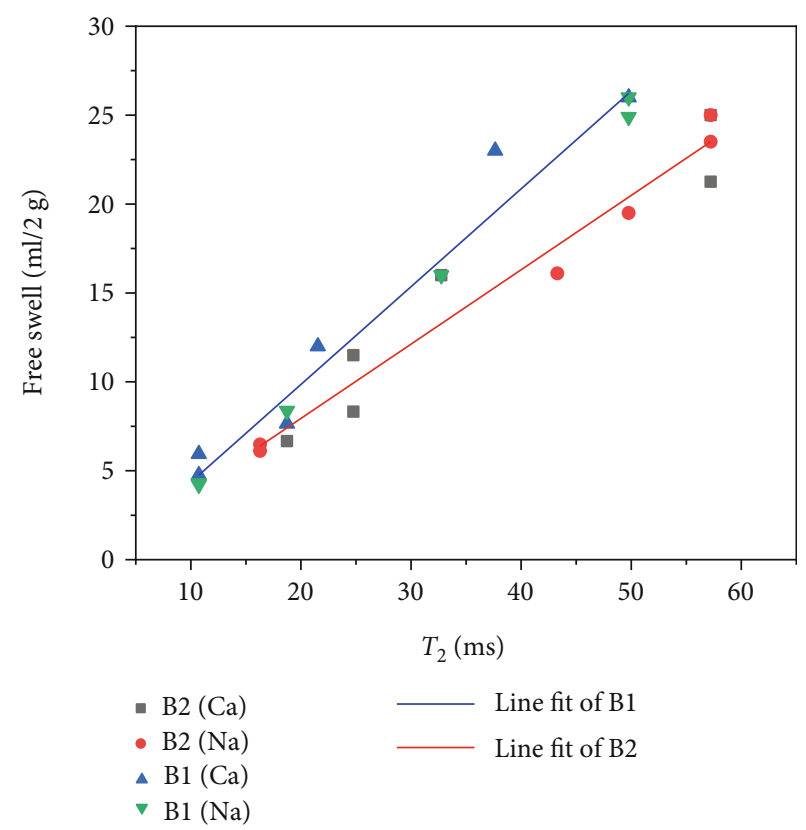

Figure 10: Free swell as a function of optimum $T_{2}$.

condition, which enhances the ability to adsorb water molecules, thereby resulting in a thicker water layer.

$$
\begin{aligned}
& \mathrm{B} 1: \mathrm{FSI}=0.41 T_{2}-0.41 R=0.96, \\
& \mathrm{~B} 2: \mathrm{FSI}=0.55 T_{2}-1.11 \quad R=0.98,
\end{aligned}
$$

4.4. Discussion of Modification Methods. Use of chemically modified bentonite appears as an alternative method to reduce the infiltration risk of the multispecies leachate. Various types of manufacturing processes were developed to reinforce the properties of Ca-bentonite such as hydraulic conductivity, swelling ability, and chemical resistance. These properties were enhanced by adding modifier to achieve the same hydraulic behavior as sodium bentonite with water [28]. As the modifier is divided into inorganic 
and organic, the mechanism of sodium modification on bentonite is also different.

The method of inorganic modification is mainly achieved by ion exchange, using the inorganic sodium salt (such as $\mathrm{Na}_{2} \mathrm{CO}_{3}$ ), and the $\mathrm{Ca}^{2+}$ replaced by ions will be stripped out of the bentonite in the form of precipitation. The precipitation $\mathrm{CaCO}_{3}$ was produced by sodium modification with $\mathrm{Na}_{2} \mathrm{CO}_{3}$ on Ca-bentonite, which turns out to be fine calcium carbonate particles that have a certain activity. Reverse reaction occurs under certain conditions, such as the presence of $\mathrm{CO}_{2}$ or a slightly lower $\mathrm{pH}$ value. The calcium ions exchange sodium ions and reenter the montmorillonite layers, so the sodium ions between the artificially sodium-modified bentonite layers are not stable.

The polymer modification mechanism is that the modifier polymers with special functional groups enter the montmorillonite layer and form layered structures such as intercalation and exfoliation. Many researchers [31-33] have conducted experimental studies on polymer-modified bentonite for antiseepage performance, and the latest experimental results have been applied to vertical cutoff walls for antiseepage performance [34]. The mechanism for enhancing antiseepage performance is as follows: (1) The intercalation of polymer into the clay mineral layer enhances the swelling of bentonite caused by chemical osmotic pressure. The reason why organic cations have a strong affinity for the clay mineral interlayer domain is that in addition to the electrostatic effect, the larger molecular weight of the organic cations, which leads to a larger van der Waals force, is also an important factor [35]. (2) The hydrogel eluted from bentonite-polymer composite can be a three-dimensional network structure between the bentonite particles, changing the microstructure and pore distribution of the soil, and blocking the cation exchange between the bentonite and external solution under the effect of wrap [36]. Liu [37] confirmed the intercalation structure of modified bentonite adding polyacrylamide from the XRD results and further proved that the modified bentonite material has a three-dimensional cross-linking network structure from the SEM image. For the modification mechanism of ionic polyacrylamide, the ionic polymer can enter the interlayer through intercalation reaction because it has a greater affinity for the interlayer domain. The reason is not only the effect of electrostatic force but also the large molecular weight and the increase of the interlayer domain of bentonite after intercalation. When the bulk density of the loaded ionic polymer increases, a cross-linked structure is also formed, which leads to greater hydration swelling capacity. In summary, the difference in the mechanism of the two modification methods leads to the difference in the properties of the modified Ca-bentonite.

\section{Conclusions}

In this study, by combining the FSI test with NMR technique, the variation law of FSI, pore water content, and hydration state of modified Ca-bentonite under the effect of various chemical solutions (different cationic valence and ionic strength) are investigated. Based on the results obtained from this study, the following conclusions can be drawn: (i) Sodium modification and polymer modification can significantly increase the FSI of Ca-bentonite. The FSI of modified Ca-bentonite in DI water is close to that of natural Na-bentonite. Under the effect of the chemical solution, the FSI of modified bentonite decreases with the increase of cation valence and ionic strength. At the same ionic strength, the effect of divalent cations on the FSI of polymer-modified Ca-bentonite is significantly greater than that of monovalent cations, but the difference on the FSI of sodium-modified Ca-bentonite is not obvious between divalent and monovalent cations. The chemical stability of polymer-modified bentonite is significantly higher than that of sodium-modified bentonite, but still less than that of natural $\mathrm{Na}$ bentonite

(ii) The $T_{2}$ distribution curves of the two kinds of modified bentonite are three-peak curves, which correspond to three types of water: surface hydration water (tightly bound water), permeated hydration water (loosely bound water), and free water. Permeated hydration water is the main form of water in bentonite, accounting for $69 \%$ to $95 \%$. With the increase of ionic strength, the content of total water and permeated hydrated water in bentonite decreases gradually, whereas the surface hydration water (accounting for $2 \%-31 \%$ ) and free water content (accounting for $0 \%-15 \%$ ) increase

(iii) Under different working conditions (different cation valence and ionic strength of chemical solutions), a uniform linear relationship exists between the FSI of different types of bentonite and the corresponding total peak area of NMR (in which the correlation coefficient is greater than 0.99). The optimal $T_{2}$ can be used to evaluate the average spacing of bentonite particles. For the same type of bentonite, a linear relationship exists between FSI and the $T_{2}$ under different working conditions (in which the correlation coefficient is greater than 0.96 )

The research on modification of clay minerals is applicable in various fields, and polymer modification attracts considerable attention. For example, the modified bentonite is used in water treatment to absorb organic pollutants in water, and the modified clay is used to block the migration of pollutants as well as solidify and stabilize heavy metal pollutants in EBSs [38-41]. Organic polymer modification of bentonite can increase the adsorption of organic pollutants, which has a better effect than inorganic modified materials [42]. The widespread application of bentonite is due to its low hydraulic conductivity and high swelling capacity, which is affected by the types of hydration state. Few studies have quantitatively evaluated the changes in various water types in hydration bentonite. The results obtained in this study provide theoretical references for the application of polymer-modified bentonite, such as inhibiting the hydration of bentonite in drilling engineering or obtaining the 
modified bentonite with the best hydration effect in impervious engineering. The modification and effect of polymer on the mineral materials studied are necessary for the polymer development and application.

\section{Data Availability}

Some or all data, models, or code that support the findings of this study are available from the corresponding author upon reasonable request.

\section{Conflicts of Interest}

The authors declare that they have no conflicts of interest.

\section{Acknowledgments}

This research was supported by the Special Fund for Basic Research on Scientific Instruments of the National Natural Science Foundation of China (grant numbers 51979267, 51625903, 51509245, and 41772342), the National Key Research and Development Program (grant number 2019YFC1804002), and the Wuhan Science and Technology Conversion Special Project (2018060403011348).

\section{References}

[1] A. K. Mishra, M. Ohtsubo, L. Li, and T. Higashi, "Controlling factors of the swelling of various bentonites and their correlations with the hydraulic conductivity of soil-bentonite mixtures," Applied Clay Science, vol. 52, no. 1-2, pp. 78-84, 2011.

[2] H. Xu, W. Zhu, X. Qian, S. Wang, and X. Fan, "Studies on hydraulic conductivity and compressibility of backfills for soil-bentonite cutoff walls," Applied Clay Science, vol. 132, pp. 326-326, 2016.

[3] A. B. A. W. Gates, Overview of Performance Compatibility Issues of GCLs with Respect to Leachates of Extreme Chemistry, 2014.

[4] J. K. Mitchell and K. Soga, Fundamentals of Soil Behavior, John Wiley and Sons Inc., New York, NY, 2005.

[5] R. J. Petrov and R. K. Rowe, "Geosynthetic clay liner (GCL) chemical compatibility by hydraulic conductivity testing and factors impacting its performance," Canadian Geotechnical Journal, vol. 863, 1997.

[6] S. L. Bradshaw, C. H. Benson, and J. Scalia, "Hydration and cation exchange during subgrade hydration and effect on hydraulic conductivity of geosynthetic clay liners," Journal of Geotechnical and Geoenvironmental Engineering, vol. 139, no. 4, pp. 526-538, 2013.

[7] C. D. Shackelford, C. H. Benson, T. Katsumi, T. B. Edil, and L. Lin, "Evaluating the hydraulic conductivity of GCLs permeated with non-standard liquids," Geotextiles and Geomembranes, vol. 18, no. 2-4, pp. 133-161, 2000.

[8] H. Y. Jo, C. H. Benson, C. D. Shackelford, J. Lee, and T. B. Edil, "Long-term hydraulic conductivity of a geosynthetic clay liner permeated with inorganic salt solutions," Journal of Geotechnical and Geoenvironmental Engineering, vol. 131, no. 4, pp. 405-417, 2005.

[9] J. Lee and C. D. Shackelford, "Impact of bentonite quality on hydraulic conductivity of geosynthetic clay liners," Journal of
Geotechnical and Geoenvironmental Engineering, vol. 131, no. 1, pp. 64-77, 2005.

[10] C. Di Maio, "Exposure of bentonite to salt solution: osmotic and mechanical effects," Géotechnique, vol. 46, no. 4, pp. 695-707, 1996.

[11] D. C. Kolstad, C. H. Benson, and T. B. Edil, "Hydraulic conductivity and swell of nonprehydrated geosynthetic clay liners permeated with multispecies inorganic solutions," Journal of Geotechnical and Geoenvironmental Engineering, vol. 130, no. 12, pp. 1236-1249, 2004.

[12] H. Y. Jo, T. Katsumi, C. H. Benson, and T. B. Edil, "Hydraulic conductivity and swelling of nonprehydrated GCLs permeated with single-species salt solutions," Journal of Geotechnical and Geoenvironmental Engineering, vol. 127, no. 7, pp. 557-567, 2001.

[13] J. Scalia and C. H. Benson, "Hydraulic conductivity of geosynthetic clay liners exhumed from landfill final covers with composite barriers," Journal of Geotechnical and Geoenvironmental Engineering, vol. 137, no. 1, pp. 1-13, 2011.

[14] P. F. Low, "Structural component of the swelling pressure of clays," Langmuir, vol. 3, no. 1, pp. 18-25, 1987.

[15] G. Xie, Y. Xiao, M. Deng et al., "Quantitative investigation of the hydration behavior of sodium montmorillonite by thermogravimetric analysis and low-field nuclear magnetic resonance," Energ Fuel, vol. 33, no. 9, pp. 9067-9073, 2019.

[16] M. MOJID and H. CHO, "Estimating the fully developed diffuse double layer thickness from the bulk electrical conductivity in clay," Applied Clay Science, vol. 33, no. 3-4, pp. 278-286, 2006.

[17] R. K. Schofield, "Calculation of surface areas from measurements of negative adsorption," Nature, vol. 160, no. 4064, pp. 408-410, 1947.

[18] H. Sun, D. Mašín, J. Najser, V. Neděla, and E. Navrátilová, "Bentonite microstructure and saturation evolution in wetting-drying cycles evaluated using ESEM, MIP and WRC measurements," Géotechnique, vol. 69, no. 8, pp. 713-726, 2019.

[19] T. Kozlowski, "Low temperature exothermic effects on cooling of homoionic clays," Cold Regions Science and Technology, vol. 68, no. 3, pp. 139-149, 2011.

[20] A. Fabbri, T. Fen-Chong, and O. Coussy, "Dielectric capacity, liquid water content, and pore structure of thawing-freezing materials," Cold Regions Science and Technology, vol. 44, no. 1, pp. 52-66, 2006.

[21] H. Wang, T. Shibue, and H. Komine, "Hydration and dehydration of water of bentonite: a solid-state $1 \mathrm{H}$ magic-angle spinning NMR study," Chemical Physics, vol. 536, p. 110796, 2020.

[22] T. Ohkubo, H. Kikuchi, and M. Yamaguchi, "An approach of NMR relaxometry for understanding water in saturated compacted bentonite," Physics and Chemistry of the Earth, Parts $A / B / C$, vol. 33, pp. S169-S176, 2008.

[23] H. Tian, C. Wei, and R. Yan, "Thermal and saline effect on mineral-water interactions in compacted clays: a NMR-based study," Applied Clay Science, vol. 170, pp. 106-113, 2019.

[24] H. Tian, C. Wei, H. Wei, R. Yan, and P. Chen, "An NMRbased analysis of soil-water characteristics," Applied Magnetic Resonance, vol. 45, no. 1, pp. 49-61, 2014.

[25] Y. Yang, K. R. Reddy, Y. Du, and R. Fan, "Sodium hexametaphosphate (SHMP)-amended calcium bentonite for slurry trench cutoff walls: workability and microstructure 
characteristics," Canadian Geotechnical Journal, vol. 55, no. 4, pp. 528-537, 2018.

[26] ASTM, Standard Test Method for Swell Index of Clay Mineral Component of Geosynthetic Clay Liners 1. D5890-11, West Conshohocken, PA, USA, 2011.

[27] S. Gao, W. G. Chapman, and W. House, "Application of low field NMR T2 measurements to clathrate hydrates," Journal of Magnetic Resonance, vol. 197, no. 2, pp. 208-212, 2009.

[28] K. Sato, G. Barast, A. R. Razakamanantsoa, I. Djeran-Maigre, T. Katsumi, and D. Levacher, "Comparison of prehydration and polymer adding effects on $\mathrm{Na}$ activated Ca-bentonite by free swell index test," Applied Clay Science, vol. 142, pp. 6980, 2017.

[29] G. Sposito, The Chemistry of Soils, Oxford University Press, New York, 2008.

[30] H. Yu, D. Sun, and C. Wei, "Swelling characteristics of bentonite with different initial water contents saturated by $\mathrm{NaCl}$ solution," Chinese Journal of Geotechnical Engineering, vol. 41, p. 595, 2019.

[31] K. Tian, C. H. Benson, and W. J. Likos, Effect of an anion ratio on the hydraulic conductivity of a bentonite-polymer geosynthetic clay liner, 2017.

[32] M. S. Hosney and R. K. Rowe, "Performance of polymerenhanced bentonite-sand mixture for covering arsenic-rich gold mine tailings for up to 4 years," Canadian Geotechnical Journal, vol. 54, no. 4, pp. 588-599, 2017.

[33] T. Katsumi, H. Ishimori, M. Onikata, and R. Fukagawa, "Longterm barrier performance of modified bentonite materials against sodium and calcium permeant solutions," Geotextiles and Geomembranes, vol. 26, no. 1, pp. 14-30, 2008.

[34] A. Norris, G. Di Emidio, M. A. Malusis, and M. Replogle, "Modified bentonites for soil-bentonite cutoff wall applications with hard mix water," Applied Clay Science, vol. 158, pp. 226-235, 2018.

[35] P. Wu, Clay Mineral Materials and Environmental Remediation, Chemical Industry Press, Beijing, 2004.

[36] J. Scalia and C. H. Benson, "Polymer fouling and hydraulic conductivity of mixtures of sodium bentonite and a bentonite-polymer composite," The Journal of Geotechnical and Geoenvironmental Engineering, vol. 143, 2017.

[37] X. Liu, Study on New Type of Polyacrylamide Modified Bentonite Impermeable Materials, Northeastern University, 2010.

[38] M. Tanyol, N. Kavak, and G. Torğut, "Synthesis of poly(ANco-VP)/zeolite composite and its application for the removal of brilliant green by adsorption process: kinetics, isotherms, and experimental design," Advances in Polymer Technology, vol. 2019, 12 pages, 2019.

[39] G. Dai, Y. Sheng, Y. Pan, G. Shi, and S. Li, "Application of a bentonite slurry modified by polyvinyl alcohol in the cutoff of a landfill," Advances in Civil Engineering, vol. 2020, 9 pages, 2020.

[40] M. M. M. Taha, C. P. Feng, and S. H. S. Ahmed, "Influence of polypropylene fibre (PF) reinforcement on mechanical properties of clay soil," Advances in Polymer Technology, vol. 2020, 15 pages, 2020.
[41] N. L. My Linh, D. Hoang Van, T. Duong, M. X. Tinh, and K. D. Quang, "Adsorption of arsenate from aqueous solution onto modified Vietnamese bentonite," Advances in Materials Science and Engineering, vol. 2019, 13 pages, 2019.

[42] Q. Zhao, H. Choo, A. Bhatt, S. E. Burns, and B. Bate, "Review of the fundamental geochemical and physical behaviors of organoclays in barrier applications," Applied Clay Science, vol. 142, pp. 2-20, 2017. 